\title{
The analgesic efficacy compared ultrasound-guided continuous transverse abdominis plane block with epidural analgesia following abdominal surgery: a systematic review and meta-analysis of randomized controlled trials
}

Chaosheng Qin ${ }^{1}$, Yuanming Liu², Jijun Xiong ${ }^{1}$, Xiaogang Wang ${ }^{1}$, Qinghua Dong ${ }^{1}$, Tingshi Su ${ }^{3}$ and Jingchen Liu ${ }^{1 *}$ (D)

\begin{abstract}
Background: This review and meta-analysis aims to evaluate the analgesic efficacy of continuous transversus abdominis plane (TAP) block compared with epidural analgesia (EA) in adults after abdominal surgery.

Methods: The databases PubMed, Embase and Cochrane Central Register were searched from inception to June 2019 for all available randomized controlled trials (RCTs) that evaluated the analgesic efficacy of continuous TAP block compared with EA after abdominal surgery. The weighted mean differences (WMDs) were estimates for continuous variables with a 95\% confidence interval $(\mathrm{Cl})$ and risk ratio (RR) for dichotomous data. The pre-specified primary outcome was the dynamic pain scores $24 \mathrm{~h}$ postoperatively.
\end{abstract}

Results: Eight trials including 453 patients (TAP block:224 patients; EA: 229 patients) ultimately met the inclusion criteria and seven trials were included in the meta-analysis. Dynamic pain scores after $24 \mathrm{~h}$ were equivalent between TAP block and EA groups (WMD:0.44; 95\% Cl: 0.1 to $0.99 ; I^{2}=91 \% ; p=0.11$ ). The analysis showed a significant difference between the subgroups according to regularly administering (4 trials; WMD:-0.11; $95 \%$ Cl: 0.32 to $0.09 ; I^{2}=0 \% ; p=0.28$ ) non-steroidal anti-inflammatory drugs (NSAIDs) or not (3 trials; WMD:1.02; $95 \%$ Cl: 0.09 to $1.96 ; I^{2}=94 \% ; p=0.03$ ) for adjuvant analgesics postoperatively. The measured time of the urinary catheter removal in the TAP group was significantly shorter ( 3 trials, WMD:-18.95, 95\% Cl:-25.22 to $-12.71 ; 1^{2}=0 \% ; p<0.01$ ), as was time to first ambulation postoperatively ( 4 trials, WMD:-6.61, 95\% Cl: -13.03 to $-0.19 ; I^{2}=67 \% ; p<0.05$ ).

Conclusion: Continuous TAP block, combined with NSAIDs, can provide non-inferior dynamic analgesia efficacy compared with EA in postoperative pain management after abdominal surgery. In addition, continuous TAP block is associated with fewer postoperative side effects.

Keywords: TAP block, Epidural analgesia, Abdominal surgery, Meta-analysis

\footnotetext{
* Correspondence: 44483316@qq.com

'Department of Anesthesiology, The First Affiliated Hospital of Guangxi Medical University, Nanning, Guangxi 530021, People's Republic of China

Full list of author information is available at the end of the article
}

C C The Author(s). 2020 Open Access This article is licensed under a Creative Commons Attribution 4.0 International License, which permits use, sharing, adaptation, distribution and reproduction in any medium or format, as long as you give appropriate credit to the original author(s) and the source, provide a link to the Creative Commons licence, and indicate if changes were made. The images or other third party material in this article are included in the article's Creative Commons licence, unless indicated otherwise in a credit line to the material. If material is not included in the article's Creative Commons licence and your intended use is not permitted by statutory regulation or exceeds the permitted use, you will need to obtain permission directly from the copyright holder. To view a copy of this licence, visit http://creativecommons.org/licenses/by/4.0/. The Creative Commons Public Domain Dedication waiver (http://creativecommons.org/publicdomain/zero/1.0/) applies to the data made available in this article, unless otherwise stated in a credit line to the data. 


\section{Background}

Epidural analgesia (EA) has long been recognized as the gold-standard technique for analgesia after abdominal surgery [1]. However, the benefits of EA are accompanied by a number of potential side effects, such as hypotension and urinary retention, which has led professionals to seek other analgesic methods $[2,3]$.

The transversus abdominis plane (TAP) block provides an analgesic effect on the anterolateral abdominal wall $[4,5]$. Growing evidence supports the effectiveness of TAP blocks for various types of abdominal surgeries. In addition, with the advancements in ultrasound technology, the safety of TAP block has greatly improved; there has been a surge of interest in ultrasound-guided TAP blocks as an adjunct for analgesia following abdominal operations $[6,7]$. However, the effect of a single TAP block is not durable, and its analgesic efficacy lasts less than $24 \mathrm{~h}[5,8]$. Thus, continuous TAP block by placing the catheter into the transverse abdominal plane and infusing local anaesthetic drugs continuously or intermittently through the catheter were used [9-11]. Continuous infusion of different doses of local anaesthetics in different regions of the TAP is complicated, and researchers have reported different and even conflicting outcomes compared with EA [12-15]. However, there has been no systematic assessment comparing the analgesic effect of continuous TAP block with traditional EA following several abdominal surgeries. Therefore, this review and meta-analysis aimed to systematically evaluate the analgesic efficacy of continuous TAP block compared with EA in adults after abdominal surgery, as well as its clinical safety and its impact on patient recovery.

\section{Methods}

\section{Search strategy and selection criteria}

We used the recommendations of PRISMA for this systematic review and meta-analysis. We searched the online databases PubMed, Embase and Cochrane Central Register for all relevant studies. Search terms included: epidural anaesthesia OR epidural analgesia OR epidural injection OR epidural administration. The results of this search subsequently combined the following terms: continuous TAP block OR continuous transversus abdominis plane block OR transversus abdominis plane catheters OR TAP block catheters OR abdominal wall block OR transversus abdominal wall block OR nerve block. The search strategy was limited to randomized controlled trials (RCTs) and those performed on humans. No language restriction was applied. The most recent electronic search was completed in June of 2019. We also manually checked the bibliographies of relevant articles for other potentially eligible trials.

\section{Population}

This systematic review and meta-analysis is only aimed at female and male adults (18 years or older) who have undergone different types of abdominal surgery.

\section{Intervention and control}

Ultrasound-guided continuous TAP blocks adopting various approaches (subcostal, oblique subcostal, lateral, or posterior $[5,16])$ compared with EA following abdominal surgeries were included in this study.

\section{Outcomes}

The pre-specified primary outcome was dynamic pain scores (upon movement) $24 \mathrm{~h}$ after abdominal surgery. Secondary outcomes were pain scores at rest after $24 \mathrm{~h}$ and pain scores, at rest and dynamic, after $12 \mathrm{~h}, 48 \mathrm{~h}$ and $72 \mathrm{~h}$. Postoperative opioid consumption was measured at $24 \mathrm{~h}, 48 \mathrm{~h}$, and $72 \mathrm{~h}$ following surgery. Meanwhile, we addressed function-related outcomes including time of removal of the urinary catheter, time to first flatus, time to first ambulation, and length of hospital stay. Outcomes of side effects were also evaluated, including hypotension and block complications within the first $24 \mathrm{~h}$ postoperatively.

\section{Data extraction}

We extracted independent data using established standard data collection forms by two authors (QCS and LYM). Disagreements were resolved by discussion with another author (LJC). If needed, we contacted the corresponding authors of selected articles to obtain the mean and standard deviation of the data. If there was no response, we used the median and quartile ranges to approximate the estimation [17, 18]. Different pain scores assessed with verbal, visual, or numeric rating scales were all converted to a standardized number (on a 10-point scale) for analysis. All opioid analgesic drug usages were converted to equianalgesic doses of intravenous morphine for quantitative evaluations $(10 \mathrm{mg}$ of IV morphine $=1.5 \mathrm{mg}$ of IV hydromorphone $=0.1 \mathrm{mg}$ of IV fentanyl $=75 \mathrm{mg}$ of IV pethidine $=100 \mathrm{mg}$ of $\mathrm{IV}$ tramadol $=30 \mathrm{mg}$ of oral morphine $=7.5 \mathrm{mg}$ of oral hydromorphone $=20 \mathrm{mg}$ of oral oxycodone) [19].

\section{Assessment of trial quality}

The quality of the reviewed trials was assessed independently by two authors (QCS and LYM) following the Cochrane Collaboration Risk of Bias Tool for randomized controlled trials [20]. Disagreements were resolved by discussion with another author (LJC). The Cochrane Risk of Bias Tool measured the following: adequacy of sequence generation, allocation concealment, blinding of participants, blinding of outcome assessment, incomplete 
outcome data, selective outcome reporting, and other potential sources of bias.

\section{Statistical analysis}

All statistical analyses were performed with the assistance of Review Manager software (RevMan version 5.3.5; The Cochrane Collaboration 2014). For continuous data, when measuring methods were different, the standardized mean difference (SMD) with $95 \%$ confidence interval (CI) was calculated; otherwise, the weight mean difference (WMD) with 95\% CI was calculated. A risk ratio (RR) with a 95\% CI was calculated for dichotomous data. The $\mathrm{I}^{2}$ statistic, used for evaluating heterogeneity, was predefined using the following three scales: low $\left(\mathrm{I}^{2}<50 \%\right)$, moderate $\left(\mathrm{I}^{2}=50-74 \%\right)$, and high $\left(\mathrm{I}^{2}>\right.$ 75\%) [21]. We pooled outcome data using a fixed effects model in the case of low heterogeneity; otherwise, we chose a random effects model. Predetermined subgroup analysis was conducted according to the type of surgical operation (open surgery or laparoscopy), the method of local anesthetic administration (continuous or intermittent), and whether other anti-inflammatory drugs were regularly used postoperatively. A $P$-value of $<0.05$ was considered statistically significant.

\section{Results}

\section{Search results}

In total, 977 potentially eligible studies were identified through the literature search. We excluded 217 records that were duplicates and a further 738 records for other reasons. After review of the remaining 22 articles in full, 8 RCTs [13, 15, 22-27] ultimately met the inclusive criteria and 7 RCTs $[13,15,22-26]$ were included in the meta-analysis. A flowchart of this process, including the reasons for excluding studies, is shown in Fig. 1.

\section{Characteristics of trials}

Ultimately included trials in this review were published between 2011 and 2017, totaling 453 patients (224 in the TAP block group and 229 in the EA group). Six trials $[13,22-25,27]$ were published in English and the other 2 trials $[15,26]$ were published in Chinese. The detailed characteristics of the included trials ( 8 RCTs) are presented in Table 1 .

\section{Risk of bias in included studies}

According to our assessment of the Cochrane Collaboration Risk of Bias tool (Fig. 2), most trials have a high risk of bias, which is mainly related to the blindness of
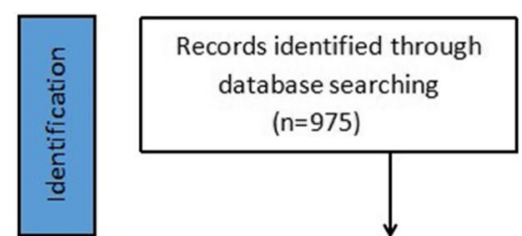

Additional records identified

through other sources $(n=2)$

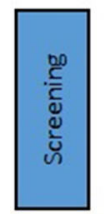

Records after duplicated removed $(n=760)$
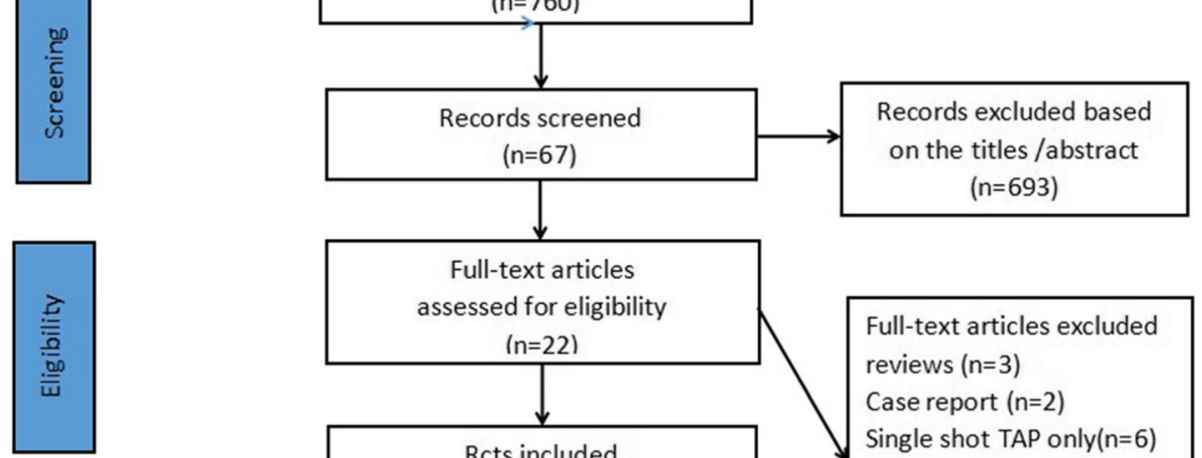

Full-text articles assessed for eligibility $(n=22)$
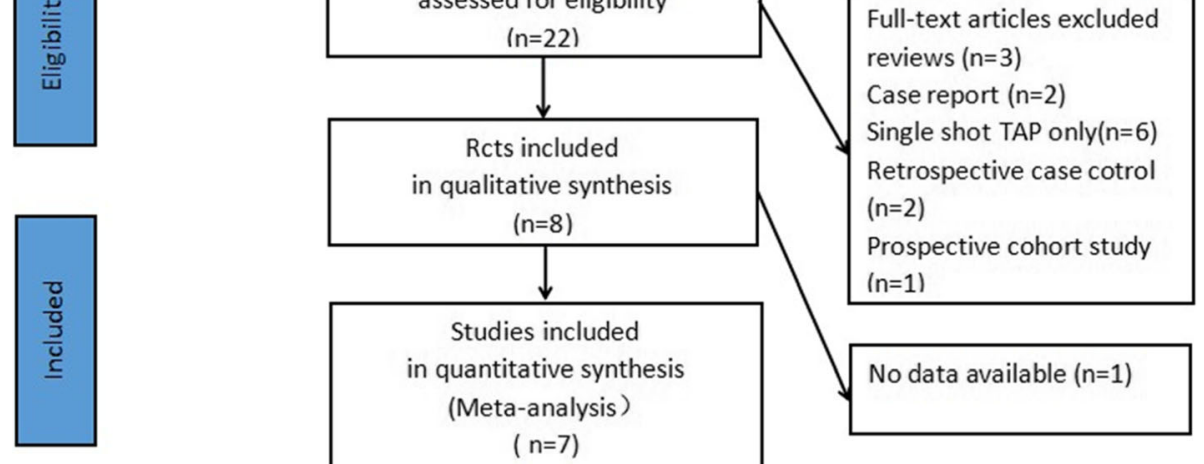

Fig. 1 Flow diagram showing results of search and reasons for exclusion of studies 


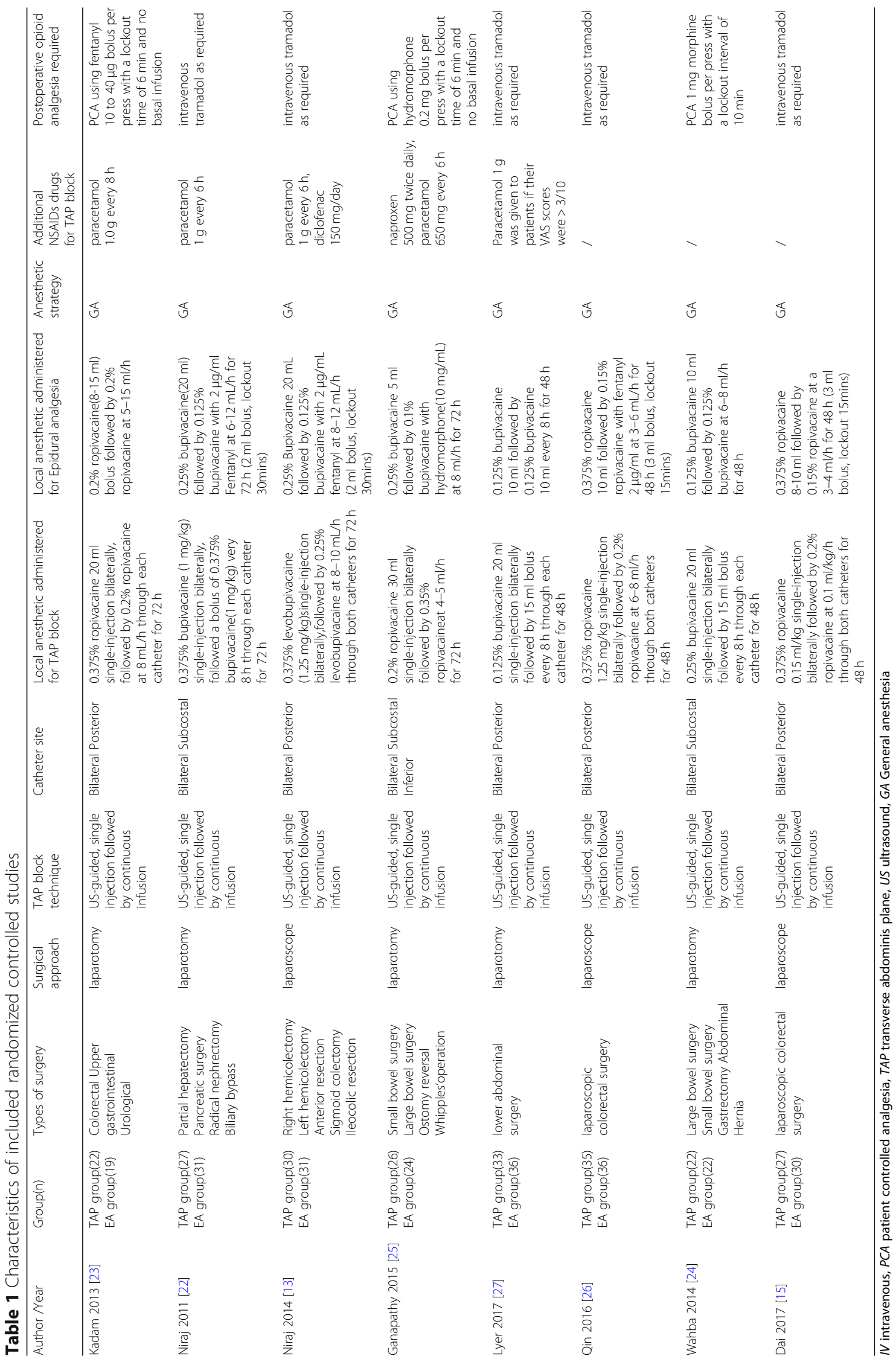




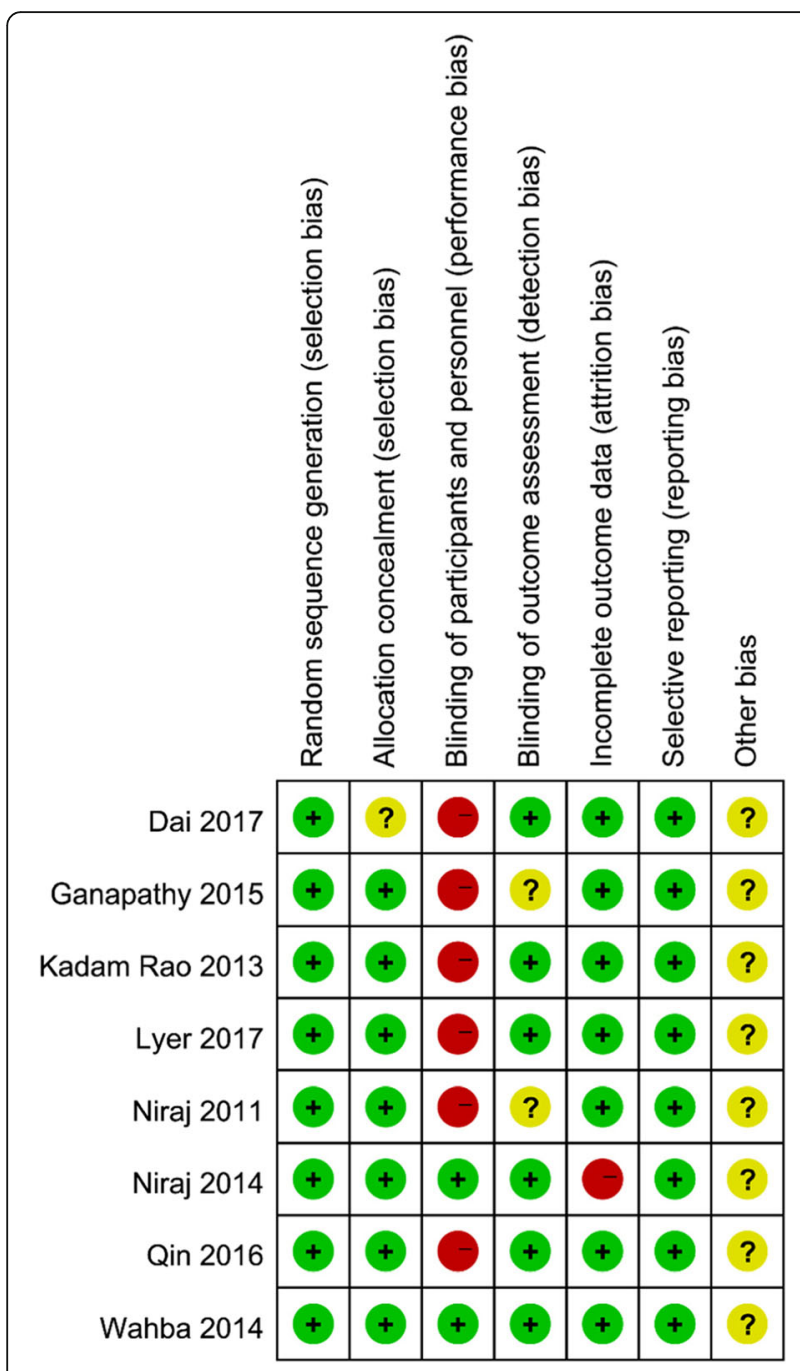

Fig. 2 Cochrane collaboration risk of bias summary: evaluation of bias risk items for each included study. Green circle = low risk of bias; red circle $=$ highrisk of bias; yellow circle $=$ unclear risk of bias

participants and evaluators. However, in these trials, it was extremely difficult to blind patients and clinicians.

\section{Primary outcome}

Seven studies $[13,15,22-26]$, including the metaanalysis with a total of 384 patients (TAP block group: 191 patients; EA group: 193 patients), reported dynamic pain scores $24 \mathrm{~h}$ postoperatively (Fig. 3). Dynamic pain scores after $24 \mathrm{~h}$ were overall equivalent between TAP block and EA groups (7 trials [13, 15, 22-26]; WMD: 0.44; $95 \% \mathrm{CI}: 0.1$ to $0.99 ; \mathrm{I}^{2}=91 \% ; p=0.11$ ). No significant difference was found in the subgroup analysis of open surgery (4 trials [23-26]; WMD:0.58; 95\% CI: 0.52 to $1.69 ; \mathrm{I}^{2}=95 \% ; p=0.3$ ) and laparoscopic surgery (3 trials [13, 15, 26]; WMD:0.29; 95\% CI: -0.18 to 0.77 ; $\mathrm{I}^{2}=79 \% ; p=0.22$ ) (Fig. 3), and no difference was found between the continuous local anesthetics subgroup (5 trials $[13,15,23,25,26]$; WMD:0.23; 95\% CI: -0.1 to $\left.0.57 ; \mathrm{I}^{2}=68 \% ; p=0.17\right)$ and intermittent local anesthetics subgroup (2 trials [22, 24]; WMD:0.85; 95\% CI: 1.41 to $3.31 ; \mathrm{I}^{2}=98 \% ; p=0.46$ ) (Fig. 4). However, there was a significant difference between the subgroups of regularly administering NSAIDs (4 trials [13, 22, 23, 25]; WMD:-0.11; 95\%CI: -0.32 to $0.09 ; \mathrm{I}^{2}=0 \% ; p=0.28$ ) or not (3 trials [15, 24, 26]; WMD:1.02; 95\%CI: 0.09 to 1.96; $\mathrm{I}^{2}=94 \% ; p=0.03$ ) for adjuvant analgesics postoperatively (Fig. 5). The pain score in the subgroup not administering NSAIDs was significantly higher. We also performed sensitivity analysis by omitting one study each time, which did not alter the overall combined WMD, and the pooled result was still robust $(P>0.05)$.

\section{Secondary outcomes}

The pain scores at rest showed no significant difference between the two groups $12 \mathrm{~h}$ ( 3 trials [15, 24, 26]; WMD: 0.67 ; $95 \%$ CI: -0.14 to $\left.1.48 ; \mathrm{I}^{2}=95 \% ; p=0.1\right), 24 \mathrm{~h}$ (6 trials [15, 22-26]; WMD:-0.07; 95\% CI: -0.21 to $0.06 ; \mathrm{I}^{2}=90 \%$; $p=0.3$ ), or $48 \mathrm{~h}$ (5 trials [15, 22-24, 26]; WMD:0.82; 95\% CI: -0.04 to $\left.1.21 ; \mathrm{I}^{2}=91 \% ; p=0.06\right)$ postoperatively.

With movement, there were no significant differences in pain scores $12 \mathrm{~h}$ (3 trials [15, 24, 26], WMD:0.99; 95\%CI: -0.10 to $\left.2.09 ; \mathrm{I}^{2}=92 \% ; p=0.07\right), 48 \mathrm{~h}(5$ trials [15, 22-24, 26];WMD:0.52; 95\% CI: -0.16 to 1.21 ; $\mathrm{I}^{2}=$ 91\%; $p=0.14)$, or $72 \mathrm{~h}$ (2 trials [22, 23]; WMD:-0.21; 95\% CI:-0.69 to $0.28 ; \mathrm{I}^{2}=72 \% ; p=0.4$ ) postoperatively. There was also no significant difference in morphine consumption postoperatively after $24 \mathrm{~h}$ (3 trials [23-25]; WMD:1.99; 95\%CI: -4.86 to $\left.8.84 ; \mathrm{I}^{2}=90 \% ; p=0.57\right), 48$ h (5 trials [13, 23-26]; WMD:4.12; 95\% CI: -3.13 to $11.16 ; \mathrm{I}^{2}=93 \% ; p=0.27$ ) (Fig. 6), and $72 \mathrm{~h}$ (3 trials [22, 23, 25]; WMD:7.67; 95\% CI: -3.40 to $18.73 ; \mathrm{I}^{2}=90 \%$; $p=0.17$ ) compared with the EA group.

\section{Recovery outcomes}

Regarding functional recovery, time to first flatus was no different between the two groups (5 trials $[13,15,24-$ 26]; WMD:1.57; $95 \% \mathrm{CI}:-4.7$ to $7.84 ; \mathrm{I}^{2}=52 \% ; P=$ $0.62)$. However, time of removal of the urinary catheter measured in the TAP group was significantly shorter (3 trials $[13,15,26]$, WMD:-18.95, 95\%CI:-25.22 to -12.71 ; $\left.\mathrm{I}^{2}=0 \% ; p<0.01\right)$, as was time to first ambulation postoperatively (4 trials $[13,15,24,26]$, WMD:-6.61, 95\%CI: 13.03 to $-0.19 ; \mathrm{I}^{2}=67 \% ; p<0.05$ ). Meanwhile, length of hospital stay revealed no difference between the TAP and EA groups (4 trials [13, 15, 23, 26], WMD:-0.02; $95 \%$ CI: -0.28 to $\left.0.23 ; \mathrm{I}^{2}=0 \% ; p=0.85\right)$.

\section{Complications}

The incidence of hypotension was significantly higher postoperatively in the EA group than in the TAP group (5 trials [15, 23-26]; RR: 0.16 ; $95 \% \mathrm{CI}$ : 0.06 to $0.42 ; \mathrm{I}^{2}=0 \%$; 


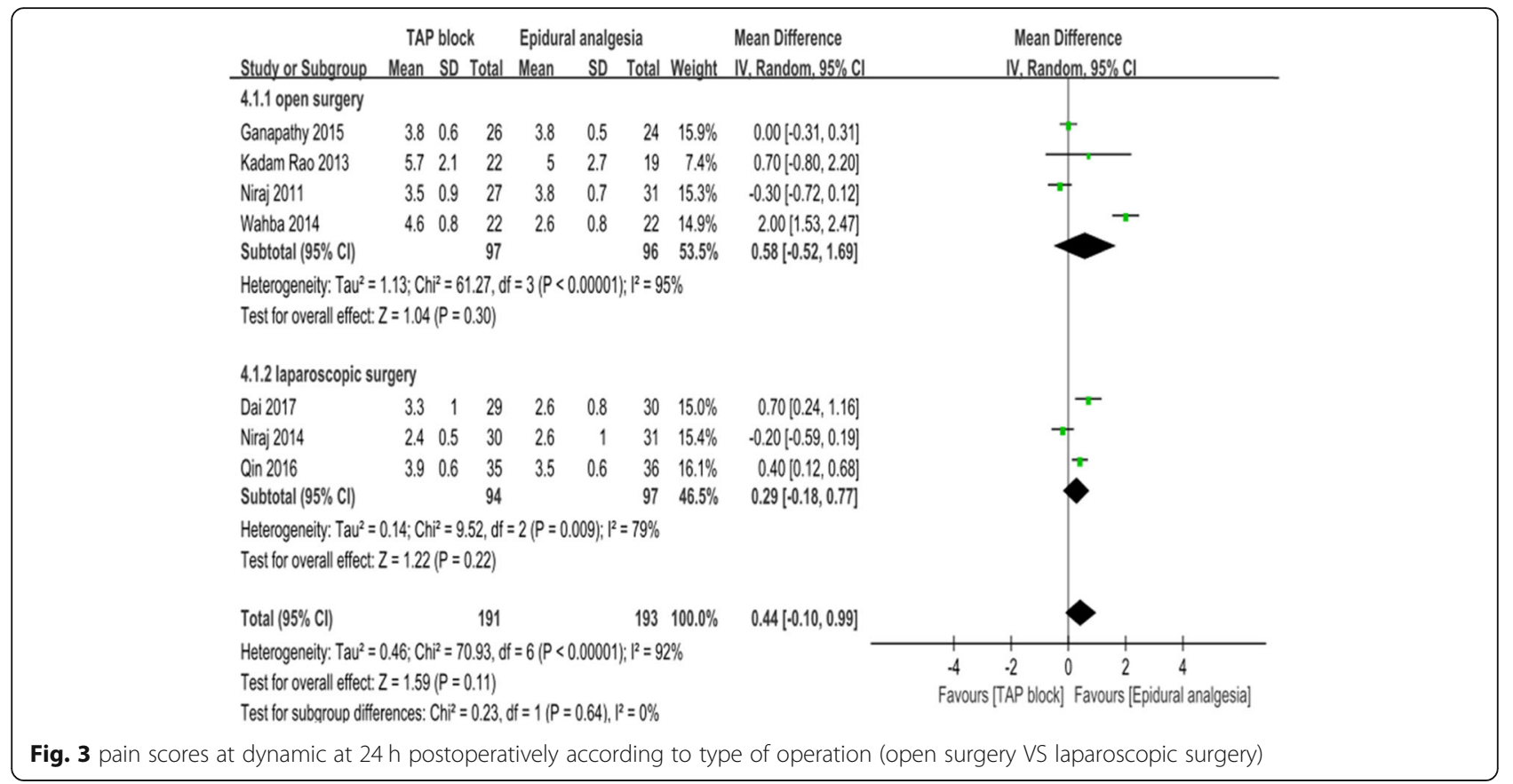

$P=0.0002)$. One trial reported that a patient in the TAP group developed a unilateral abdominal wall hematoma immediately after surgery. However, the author was unclear whether this was due to a trauma caused by the insertion of the TAP catheter or surgical puncture.

\section{Discussion}

This review and meta-analysis, comparing continuous TAP block with EA in adults after abdominal surgery, included 8 RCTs with a total of 453 patients. The result of the meta-analysis suggested no significant difference in pain scores between the two groups $24 \mathrm{~h}$ postoperatively. There was also no significant difference in pain scores postoperatively, as well as no difference in equianalgesic consumption of intravenous morphine.

The location of injection into the TAP alters the spread and effect of TAP blocks. It is proposed that the range of TAP injections be classified as follows [16]: upper subcostal TAP (deep to the rectus, mainly covering T7 and T8), lower subcostal TAP (lateral to rectus,

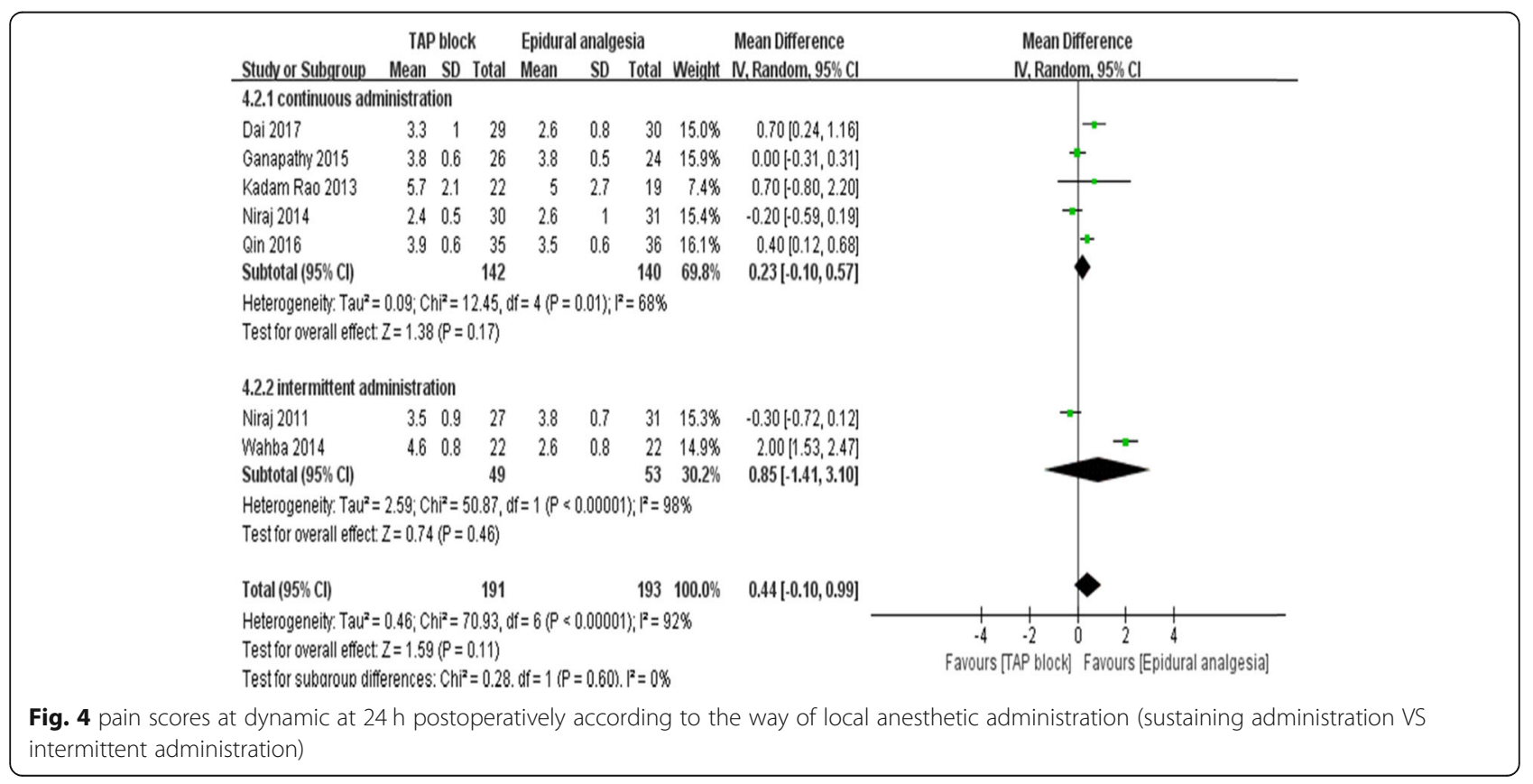




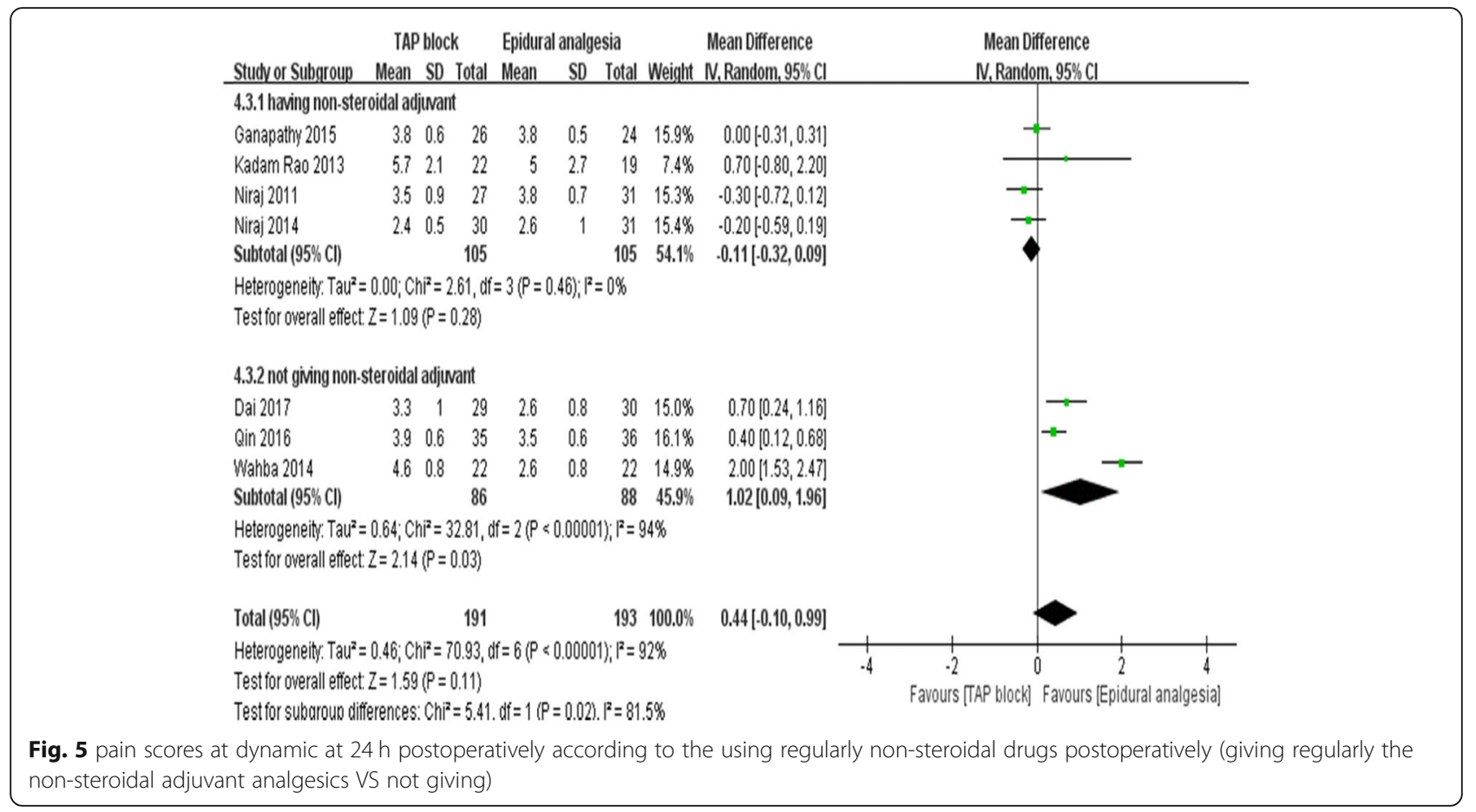

mainly covering T11), lateral TAP (midway between the costal margin and iliac crest in the mid-clavicular line, mainly covering T11 and T12), ilio-inguinal TAP (near the iliac crest lateral to the anterior superior iliac spine, mainly covering T12 and L1), and posterior TAP (in the triangle of Petit). A previous meta-analysis comparing TAP block with EA suggested that TAP block can provide equivalent analgesic effect at rest $24 \mathrm{~h}$ after abdominal surgery [28]. However, studies have shown that the analgesic effect of a single TAP block lasts less than $24 \mathrm{~h}[29,30]$.
This review included both single TAP block and continuous TAP block. For this reason, choosing a timepoint of $24 \mathrm{~h}$ after surgery as the endpoint for the primary outcome might increase the bias of analysis. Therefore, in our review, we excluded the single TAP block variable and chose the dynamic pain score at $24 \mathrm{~h}$ after surgery as our primary outcome, which can adequately reflect the efficacy and duration of continuous TAP block.

In addition, the subgroup analysis of laparotomy and laparoscopic surgery showed no significant difference,

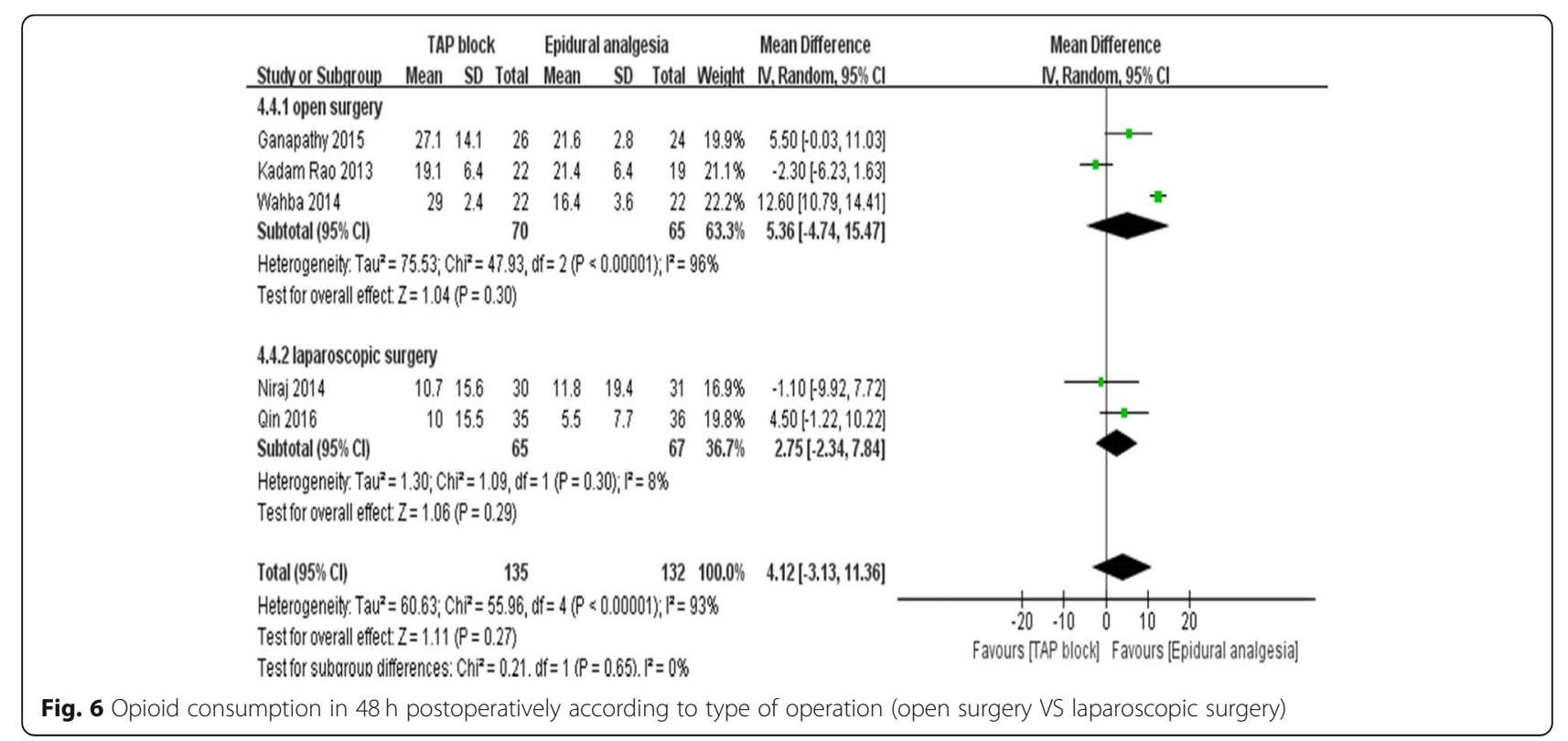


and a similar result was found in the subgroups comparing the mode of local anesthetic administration. However, the heterogeneity between trails should be noted. Laparoscopic surgery is considered minimally invasive compared with open surgery in the clinical setting $[31,32]$. Various types of abdominal surgery were included in this meta-analysis, which easily explains why the heterogeneity of the laparotomy group was significantly higher than in the laparoscopy group. According to the mode of local anesthetic administration, only two trials were included in the intermittent administration group, and there was a relatively small sample size. We also analyzed those who took NSAIDs postoperatively as a separate subgroup. However, there was a significant difference between the subgroups. Meanwhile, the heterogeneity in the NSAIDs group was significantly reduced. This suggested that TAP block combined with NSAIDs can provide more relief for patients after abdominal surgery. The NSAIDs would better treat the visceral pain and reduce the usage of opioids postoperatively [33]. Therefore, TAP block is usually combined with NSAIDs to participate in multimodal analgesia [13, 34].

Continuous TAP block analgesia does not cause urinary retention compared with EA postoperatively. On the contrary, patients who received EA used the urinary catheter for significantly longer. Due to limited reports on the outcomes of the complications between the two groups, we cannot draw more evidential results about the relative benefits of the two technologies. However, it should be noted that the episodes of postoperative hypotension associated with EA were significantly higher than those of the TAP group.

\section{Limitations}

There are several limitations that must be taken into consideration when interpreting the results of this review. Firstly, because of different surgical procedures, the location of TAP blocks and local anesthetic infusion strategies may be individualized. There are many factors that affect the procedure of continuous TAP block, including puncture location, catheter size, depth of catheter insertion, and various local anesthetic dosage, which will increase the heterogeneity between trails. Secondly, the protocols of anti-inflammatory drugs in the included trials were significantly different (including the type of drug, dosage and delivery speed), which may lead to increased heterogeneity. Such anti-inflammatory drugs may interfere with the overall evaluation of the pain score (somatic and visceral pain). Furthermore, the success of the TAP catheter also depends on the surgeon's level of experience. Moreover, it was extremely difficult to blind patients and clinicians, when we were conducting a TAP block performance, but we judge that this lack of blindness is unlikely to affect our primary outcomes. In addition, specific criteria for removing the urinary catheter and specific ambulation protocols were not defined in the same way in the included trials. Therefore, more structured and standardised continuous TAP blocking protocols should be developed to compare with EA.

\section{Conclusion}

This systematic review and meta-analysis suggests that the technique of continuous TAP block, combined with NSAIDs, can provide non-inferior dynamic analgesia efficacy compared with epidural infusion in adults after abdominal surgery. Continuous TAP block presents another option for effective and safe extended analgesia postoperatively. However, additional higher-quality RCTs would better define the comparable efficacy before supporting a stronger recommendation for continuous TAP block, which causes less hypotension and allows for a significantly shorter duration of urinary catheter use postoperatively compared with EA after abdominal surgery.

\section{Abbreviations \\ Cl: Confidence interval; EA: Epidural analgesia; NSAIDs: Non-steroidal antiinflammatory drugs; PRISMA: Preferred reporting items for systematic reviews and meta-analyses; RCTs: Randomized controlled trials; RR: Risk ratio; SMD: Standardized mean difference; TAP: Transversus abdominis plane block; VAS: Visual analogue scale; WMD: Weighted mean difference}

\section{Acknowledgements}

Not Applicable.

\section{Authors' contributions}

Study conception and design - QCS and LC; Extraction of data - QCS and LYM; interpretation and analysis of data - QCS, WXG and STS; writing manuscript - QCS, XJJ and DQH. All authors read and approved the final manuscript.

\section{Funding \\ No funding}

Availability of data and materials

All data generated or analyzed during this study are included in this published article.

\section{Ethics approval and consent to participate}

Not applicable.

\section{Consent for publication}

Not applicable.

\section{Competing interests}

The authors declare that they have no competing interests.

\section{Author details}

'Department of Anesthesiology, The First Affiliated Hospital of Guangxi Medical University, Nanning, Guangxi 530021, People's Republic of China. 2Department of Ultrasound, Affiliated Hospital of Guilin Medical University, Guilin, Guangxi 541001, People's Republic of China. ${ }^{3}$ Department of radiotherapy, Affiliated Tumor Hospital of Guangxi Medical University, Nanning, Guangxi 530021, People's Republic of China. 
Received: 2 July 2019 Accepted: 21 February 2020

Published online: 28 February 2020

\section{References}

1. Rodgers A, Walker N, Schug S, McKee A, et al. Reduction of postoperative mortality and morbidity with epidural or spinal anaesthesia: results from overview of randomised trials. BMJ. 2000;321(7275):1493.

2. Popping DM, Elia N, Van Aken HK, et al. Impact of epidural analgesia on mortality and morbidity after surgery: systematic review and meta-analysis of randomized controlled trials. Ann Surg. 2014;259(6):1056-67.

3. Bos EME, Hollmann MW, Lirk P. Safety and efficacy of epidural analgesia. Curr Opin Anaesthesiol. 2017;30(6):736-42.

4. Jakobsson J, Wickerts L, Forsberg S, et al. Transversus abdominal plane (TAP) block for postoperative pain management: a re view. F1000Res. 2015;4.

5. Tsai HC, Yoshida T, Chuang TY, Yang SF, et al. Transversus Abdominis plane block: an updated review of anatomy and techniques. Biomed Res Int. 2017:2017:8284363.

6. Chin KJ, McDonnell JG, Carvalho B, et al. Essentials of our current understanding: abdominal wall blocks. Reg Anesth Pain Med. 2017;42(2):133-83.

7. Kim AJ, Yong RJ, Urman RD. The Role of Transversus Abdominis Plane Blocks in Enhanced Recovery after Surgery Pathways for Open and Laparoscopic Colorectal Surgery. J Laparoendosc Adv Surg Tech. 2017;27(9): 909-14

8. Maeda A, Shibata SC, Kamibayashi T, et al. Continuous subcostal oblique transversus abdominis plane block provides more effective analgesia than single-shot block after gynaecological laparotomy: a randomised controlled trial. Eur J Anaesthesiol. 2015;32(7):514-5.

9. Gomez-Rios MA, Paech MJ. Continuous posterior TAP analgesia after laparoscopic colorectal surgery. Anaesthesia. 2014;69(9):1054-5.

10. Niraj G, Kelkar A, Hart E, et al. Four quadrant transversus abdominis plane block and continuous transversus abdominis plane analgesia: a 3-year prospective audit in 124 patients. J Clin Anesth. 2015;27(7):579-84.

11. Bakes D, Littlejohn C, Frenk V. Effectiveness of adding transverse abdominus plane (TAP) catheters to patient-controlled analgesia (PCA) in laparoscopic colon resections: Aretrospective chart review. Dis Colon Rectum. 2016;59(5):e310.

12. Heil JW, Nakanote KA, Madison SJ, et al. Continuous transversus abdominis plane (TAP) blocks for postoperative pain control after hernia surgery: a randomized, triple-masked, placebo-controlled study. Pain Med. 2014;15(11): 1957-64.

13. Niraj G, Kelkar A, Hart E, et al. Comparison of analgesic efficacy of fourquadrant transversus abdominis plane (TAP) block and continuous posterior TAP analgesia with epidural analgesia in patients undergoing laparoscopic colorectal surgery: an open-label, randomised, non-inferiority trial. Anaesthesia. 2014;69(4):348-55.

14. Cowlishaw PJ, Kotze PJ, Gleeson L, et al. Randomised comparison of three types of continuous anterior abdominal wall block after midline laparotomy for gynaecological oncology surgery. Anaesth Intensive Care. 2017:45(4): 453-8.

15. Dai Y, Sun XD, Liu JC. A comparative study of the analgesic effect of transverse abdominal block and thoracic epidural block after colorectal surgery. J Guanxi Med Univ. 2017;34(08):1213-6.

16. Hebbard P. TAP block nomenclature. Anaesthesia. 2015;70(1):112-3.

17. Wan X, Wang W, Liu J, et al. Estimating the sample mean and standard deviation from the sample size, median, range and/or interquartile range. BMC Med Res Methodol. 2014;14:135.

18. Collaboration, TC: Cochrane Handbook for systematic reviews of interventions version 5.1.0. Available at: http://ims.cochrane.org/revman. Accessed 20 Sept 2017.

19. Shaheen $P E$, Walsh $D$, Lasheen $W$, et al. Opioid equianalgesic tables: are they all equally dangerous? J Pain Symptom Manag. 2009;38(3):409-17.

20. Higgins JP, Altman DG, Gotzsche PC, et al. The Cochrane Collaboration's tool for assessing risk of bias in randomised trials. BMJ. 2011;343:d5928.

21. Melsen WG, Bootsma MC, Rovers MM, et al. The effects of clinical and statistical heterogeneity on the predictive values of results from metaanalyses. Clin Microbiol Infect. 2014;20(2):123-9.

22. Niraj G, Kelkar A, Jeyapalan I, et al. Comparison of analgesic efficacy of subcostal transversus abdominis plane blocks with epidural analgesia following upper abdominal surgery. Anaesthesia. 2011;66(6):465-71.

23. Rao Kadam V, Van Wijk RM, Moran Jl, et al. Epidural versus continuous transversus abdominis plane catheter technique for postoperative analgesia after abdominal surgery. Anaesth Intensive Care. 2013;41(4):476-81.
24. Wahba SS, Kamal SM. Analgesic efficacy and outcome of transversusabdominis plane block versus low thoracic-epidural analgesia after laparotomy in ischemic heart disease patients. J Anesth. 2014;28(4):517-23.

25. Ganapathy S, Sondekoppam RV, Terlecki M, et al. Comparison of efficacy and safety of lateral-to-medial continuous transversus abdominis plane block with thoracic epidural analgesia in patients undergoing abdominal surgery: a randomised, open-label feasibility study. Eur J Anaesthesiol. 2015; 32(11):797-804

26. Qin CS, Lin YN, Liu JC. Clinical study of ultrasound-guided continuous transversus abdominis plane block on thefficacy of postoperative analgesia in patients undergoing laparoscopic colorectal surgery. J Minim Invasive Med. 2016;11(05):664-8.

27. Lyer SS, Bavishi H, Mohan CV, et al. Comparison of epidural analgesia with Transversus Abdominis plane analgesia for postoperative pain relief in patients undergoing lower abdominal surgery: a prospective randomized study. Anesth Essays Res. 2017;11(3):670-5.

28. Baeriswyl M, Zeiter F, Piubellini D, et al. The analgesic efficacy of transverse abdominis plane block versus epidural analgesia: a systematic review with meta-analysis. Medicine (Baltimore). 2018;97(26):e11261.

29. Stoving $\mathrm{K}$, Rothe $\mathrm{C}$, Rosenstock CV, et al. Cutaneous sensory block area, muscle-relaxing effect, and block duration of the Transversus Abdominis plane block: a randomized, blinded, and placebo-controlled study in healthy volunteers. Reg Anesth Pain Med. 2015;40(4):355-62.

30. Abdallah FW, Laffey JG, Halpern SH, et al. Duration of analgesic effectiveness after the posterior and lateral transversus abdominis plane block techniques for transverse lower abdominal incisions: a meta-analysis. Br J Anaesth. 2013; 111(5):721-35

31. Trejo-Avila ME, Valenzuela-Salazar C, Betancourt-Ferreyra J, et al. Laparoscopic versus open surgery for abdominal trauma: a case-matched study. J laparoendosc Adv Surg Tech A. 2017;27(4):383-7.

32. Arsalani-Zadeh $\mathrm{R}, \mathrm{Ull}$ ah $\mathrm{S}$, Khan $\mathrm{S}$, et al. Oxidative stress in laparoscopic versus open abdominal surgery: a systematic review. J Surg Res. 2011;169(1): e59-68.

33. Choi YM, Byeon GJ, Park SJ, et al. Postoperative analgesic efficacy of singleshot and continuous transversus abdominis plane block after laparoscopic cholecystectomy: a randomized controlled clinical trial. J Clin Anesth. 2017; 39:146-51.

34. Jadon A, Jain P, Chakraborty S, et al. Role of ultrasound guided transversus abdominis plane block as a component of multimodal analgesic regimen for lower segment caesarean section: a randomized double blind clinical study. BMC Anesthesiol. 2018;18(1):53.

\section{Publisher's Note}

Springer Nature remains neutral with regard to jurisdictional claims in published maps and institutional affiliations.

Ready to submit your research? Choose BMC and benefit from:

- fast, convenient online submission

- thorough peer review by experienced researchers in your field

- rapid publication on acceptance

- support for research data, including large and complex data types

- gold Open Access which fosters wider collaboration and increased citations

- maximum visibility for your research: over $100 \mathrm{M}$ website views per year

At BMC, research is always in progress.

Learn more biomedcentral.com/submissions 